\title{
Endomicroscopy Image Recognition using Ensemble Neural network with Contrast Limited Adaptive Histogram Equalisation
}

\author{
Kalaivani. N, Kani Mozhi. N, Kanimozhi. M, Kalieswari. S, Kuralarasi. R
}

\begin{abstract}
Endomicroscopy is a small tool used for cancer diagnosis, this enables in-vivo imaging at microscopic resolution closely to histology image during endoscopic procedures and captured image within the dataset has high imaging quality resulting in an inequality between moral and poor-quality images. There's no clear demonstration of the artifacts in an endomicroscopy producer. During this proposed method, the ensemble neural network (ENN) approach models to scale back the variance of predictions and reduce generalization error with contrast limited adaptive histogram equalization (CLAHE) algorithm were used to recover the image pixel balancing. Binary classification of accuracy $98.79 \%$ has been achieved.

Keywords: Breast cancer, Ensemble neural network (ENN), Contrast limited adaptive histogram equalization (CLAHE).
\end{abstract}

\section{I.INTRODUCTION}

To diagnose cancer tissues by using endomicroscopy, it enables in-vivo imaging at microscopic resolution. A miniaturized optics and versatile fiber-bundle provides realtime access to histology information during clinical procedures and has established promising sensitivity and specificity in several pre-clinical and clinical, including within the digestive region, mouth, esophagus, breast, and lungs. In clinical procedures, histopathological examination of biopsy samples by trained pathologists is that the gold standard for disease diagnosis, grouping and classifying. Even though endomicroscopic enable the attainment of microscopic images that closely to histology images and reliable diagnosis is not difficult for several clinicians. The standard of study needs good performance on both mosaic and features. Endomicroscopic has a large dataset, the field of view images, and supply an accurate diagnosis by manual identification.

Revised Manuscript Received on April 25, 2020.

* Correspondence Author

Kalaivani.N*, Electronics and Communication Engineering, Sri Krishna College of Engineering and Technology, Coimbatore, India. Email: kalaivani@skcet.ac.in

KaniMozhi.N, Electronics and Communication Engineering, Sri Krishna College of Engineering and Technology, Coimbatore, India. Email: 18epcm005@skcet.ac.in

KaniMozhi.M, Electronics and Communication Engineering, Sri Krishna College of Engineering and Technology, Coimbatore, India. Email: 18epcm004@skcet.ac.in

(c) The Authors. Published by Blue Eyes Intelligence Engineering and Sciences Publication (BEIESP). This is an open access article under the CC BY-NC-ND license (http://creativecommons.org/licenses/by-nc-nd/4.0/)

\section{LITERATURE SURVEY}

Breast cancer occurs commonly in our society, and an early analysis is necessary for patients to treat and it influences an irretrievable stage. Various schemes are developing for making the process simpler for diagnosing the disease. Ensemble neural networks known as radial basis function network (RBFN), generalized regression neural network (GRNN) and feedforward neural network (FFNN) was employed for separation of carcinoma data models as normal and abnormal classes.

The conveniences of various approaches and hybrid model that is a combination of various methods was discovered and the performances of all methods were computed. Contrast enrichment plays a major part in image processing applications. A low complexity method was proposed for means of contrast enhancement. This method achieves high-frequency of a data for estimation of intensity-weighting matrix, that can be adapted for control of Gaussian fitting curve and then distribution of the contrast gain was formed. As the curve can be designed easily the reinforcement details can be unseen in notable regions. Later, the grayscale transformation that was attained from Gaussian fitting expresses the contrast dispersal. This method, is entirely automatic and the output obtained describes the occurrence of the traditional enhancement methods, particularly within the characteristics such as visual pleasure, anti-noise capability, and target-oriented contrast enhancement.

\section{EXISTING SYSTEM}

To identify medical outcomes for cancer patients, deep learning was used. To determine the typical character from the gene expression data, combination of analysis of principal component, auto encoder neural network and exportation of deep learning was used. Utilization of AdaBoost algorithm was used in the classifier learning phase to produce ensemble classifier in the final estimation. In this method, combination of feature selection and feature extraction is combined with deep learning methods to determine the typical features of gene expression to produces on efficient classifier in breast cancer estimation.

\section{A. Supervised classifier learning}

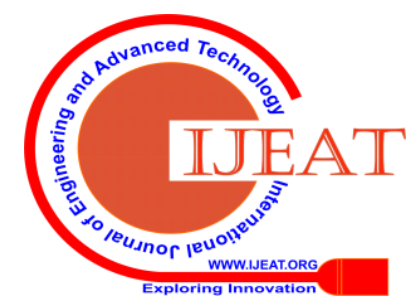


For classifier learning approach, AdaBoost algorithm that provides outstanding classification performance is used. Further, for comparison in estimation, PCA-Ada using PCA classifier is developed in contrast with classifier.

\section{B. Feature extraction}

In Feature learning approach, the stacked auto encoder is used for developing a deep neural network through assembling numerous auto encoders hierarchically.

\section{Autoencoder neural network}

The Exponential Linear Unit that increases the speeding process of the training phase in deep neural networks is used and it also leads to increased accuracy in classification process.

\section{D. classifier learning by Ada boost algorithm}

For training the classifier, a supervised algorithm called AdaBoost algorithm is used to analyze the binary classifier. It also provides efficient methods that increases the accuracy of the classification by combination of weak classifier and a strong classifier.

\section{E. Performances of ensemble classifiers}

To determine the characteristics of gene profiles and classifier, combined training assessment dataset was used, and it was performed for about 10 times five-fold cross-validation on GSE2034. For each of the process, the whole of the dataset is randomly divided into five clusters. For training process, four groups were used and another group is used in evaluation. Additionally, independent tests were performed and PCA-AE-Ada performed well and it was stable in both testing and training phase. This method achieved better results but the generalization capacity must be improved further.

\section{PROPOSED SYSTEM}

Breast cancer is one of the types of cancer and became common disease now a days. In order, to reduce the number of patients suffering from cancer disease initial detection must be performed. Initial detection of breast cancer improves survival rate by $95 \%$. In this paper, cancer is diagnosed by endomicroscopy. The captured images are closely to histology modalities and provides poor pixel balancing to improve that contrast-limited adaptive histogram equalization was used to enhance the contrast of image.

So that, easy to find normal and abnormal classes with ensemble neural network is used to reduce the generalization error and improves the accuracy and precision.

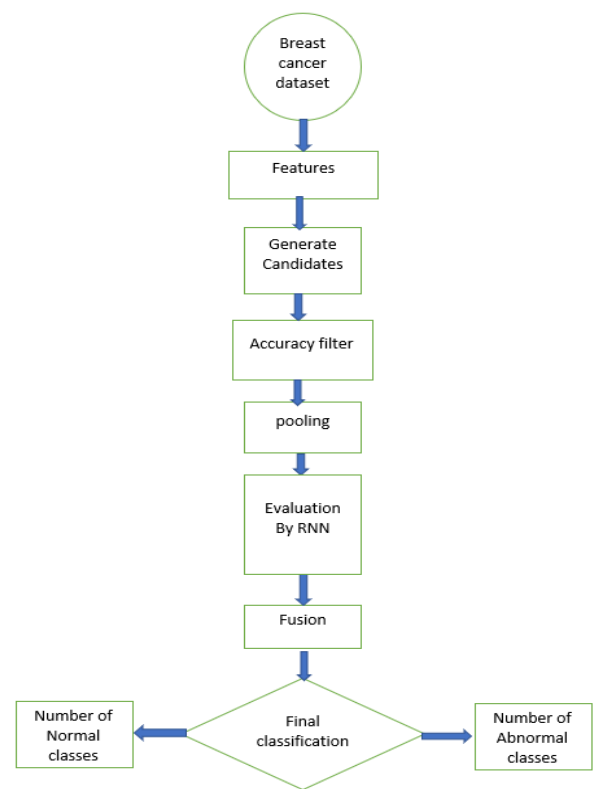

Fig.1. Overview of the proposed research methodology

\section{A. Dataset}

Breast tissue samples are attained from about 45 patients that are detected with two classes: normal and abnormal. During the completion of endomicroscopic images, each breast tissue samples undergone routine histopathology processes to get the histology pictures. The evaluation metric is described by classification accuracy. The proposed system that serves as a method for detecting the normal and abnormal classes to help the clinical field.

\section{B. Features}

These features are Abnormality Assessment Rank, Texture (grey-scale values); Edges; Smoothness; Interest points and Firmness.

\section{Generate Candidates}

The number of images that remains selected to determine the candidate pooling would cause increase in amount of ensemble neural networks.

\section{Accuracy Filter and Candidate Pooling}

In the process of training and testing phases, the classifiers having greater accuracy are carefully chosen that is meant for candidate pool. In some of the cases, same accuracy can be obtained in the selection process, in such situation's preference must be provided to the process that has the lowest amount in hidden layer.

This was carried out to guarantee a low computation rate of ensemble scheme. By utilizing the training and testing datasets experiments will be carried out.

\section{E. Fusion Strategy}

By combining each of the classifiers, ensemble relies are developed and finally results are fused. To obtain an efficient result, base classifier was chosen and it produced higher accuracy. Final step involved adding of the classifier and fusion of results that produced overall classification. The contrast limited adaptive histogram algorithm and ensemble neural network algorithm was used in the classification process to obtain higher accuracy.

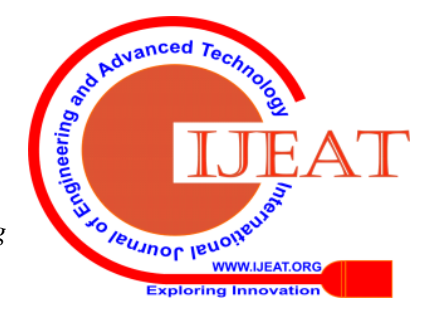




\section{F. Contrast Limited Adaptive Histogram Equalization}

To implement this method, a picture that is of a size with grey levels was taken into consideration and each of the element represented the membership of equivalent pixel with an image property. For enhancement of image pixel balancing, CLAHE algorithm was employed.

\section{G. Neural Network}

Ensemble neural networks consists of multiple feed-forward neural networks.

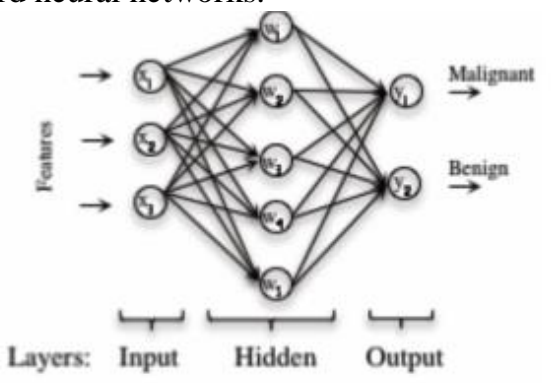

Fig.2. Ensemble neural network

A feed-forward neural network consisting of interrelated processing units ordered into several layer. The input features are represented by the primary layer and the classification is represented by the output layer. In between various other layer exists and are termed as hidden layers. The accuracy of the classification was variable.

\section{H. Confusion Metrics}

The following evaluation metrics are:

- False Positive (FP): A tested outcome indicates wrongly that the patient having cancer.

- False Negative (FN): A tested result indicates wrongly that the patient having no cancer.

- $\quad$ True Positive (TP): Predicts the positive class.

- $\quad$ True Negative (TN): Predicts the negative class.

\section{Precision}

Which is closeness of a two or more measurement values.

$$
\text { Precision }=\frac{\text { True Positives }}{\text { True Positives + False Positives }}
$$

Recall

Recall the number of relevant information that were actually retrieved.

\section{True Positives}

Recall $=$

True Positives + False Negatives

\section{F1-Score}

F-measure is the combination of precision and recall for finding harmonic mean.

$$
\text { F1 Score }=\frac{2 \times(\text { Precision } \times \text { Recall })}{\text { Precision }+ \text { Recall }}
$$

Accuracy

Which is closeness of a measured value to a standard value.

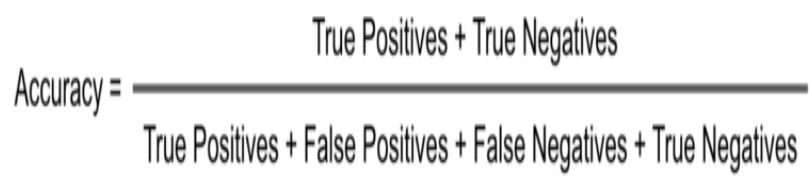

The corresponding evaluation metrics are tabulated in Table I. The results show that the proposed model improves the accuracy and sensitivity.

Table-I: Classification of accuracy, sensitivity, and specificity

\begin{tabular}{|l|l|l|c|}
\hline $\begin{array}{l}\text { Metrics / } \\
\text { Algorithms }\end{array}$ & $\begin{array}{l}\text { Accuracy } \\
\text { (\%) }\end{array}$ & $\begin{array}{l}\text { Sensitivity } \\
\text { (\%) }\end{array}$ & $\begin{array}{l}\text { Specificity } \\
\text { (\%) }\end{array}$ \\
\hline ENN+AHE & 98.78 & 1 & 90.47 \\
\hline
\end{tabular}

\section{SIMULATION RESULTS}

\section{A. Histology modalities}

The corresponded region in histology slides is shown in Fig.5.1

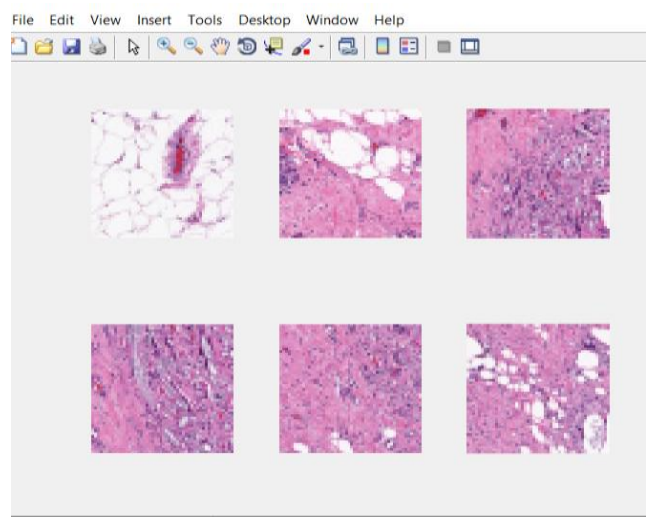

Fig.5.1 Histology modalities

\section{B. Pre-processing image}

The RGB to Grayscale image is shown in Fig.5.2

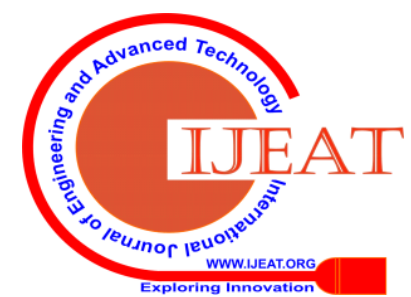



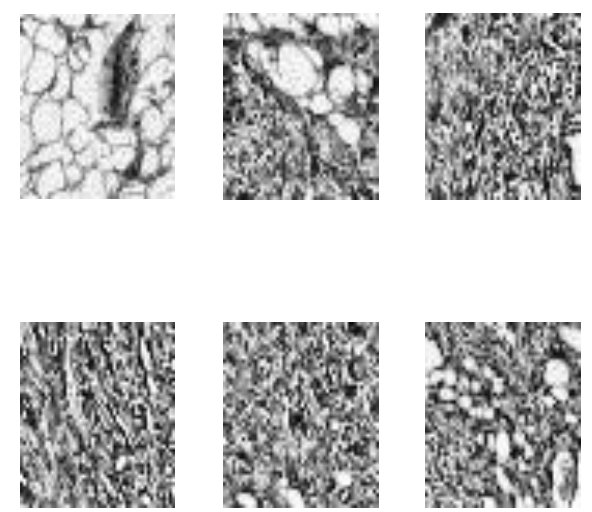

Fig.5.2 Pre-processing image

\section{Convolutional layered image}

The better quality of the contrastenhanced image is shown in Fig.5.3
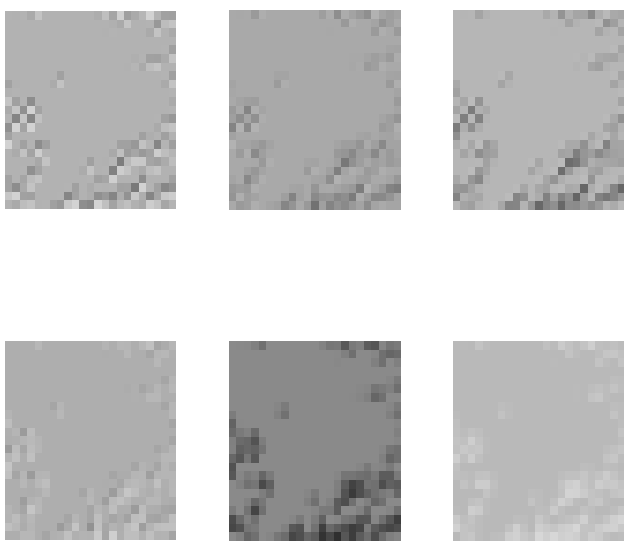

Fig.5.3 Convolutional layered image

\section{Confusion matrix}

Confusion matrix is based on tp, tn, fp and fn is shown in Fig.5.4

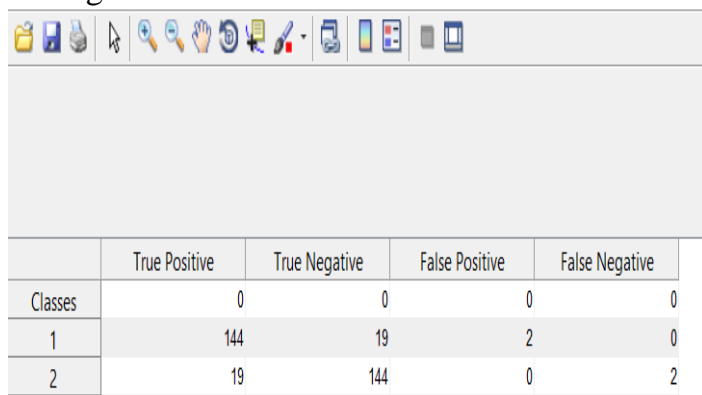

Fig.5.4 Confusion matrix

\section{E. Accuracy and precision}

The accuracy and precision values obtained from the confusion matrix is shown in Fig. 5.5
File Edit View Insert Tools Desktop Window Help

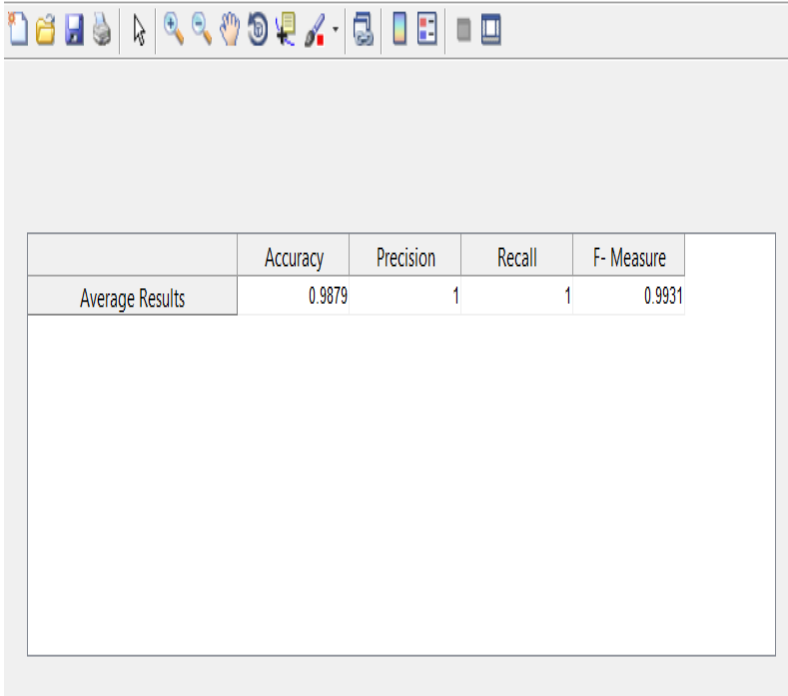

Fig.5.5 Obtained accuracy and precision

\section{F. Normal and Abnormal detection}

The output window displaying the normal and the abnormal samples is shown in Fig. 5.6

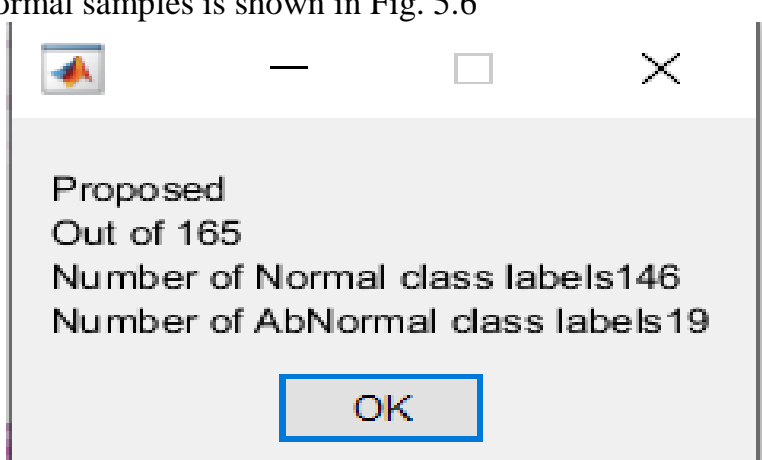

Fig.5.6 Output screen showing the number of normal class and abnormal class

\section{G. Performance Analysis}

Performance analysis of the existing system and the proposed system as shown in Fig.5.7

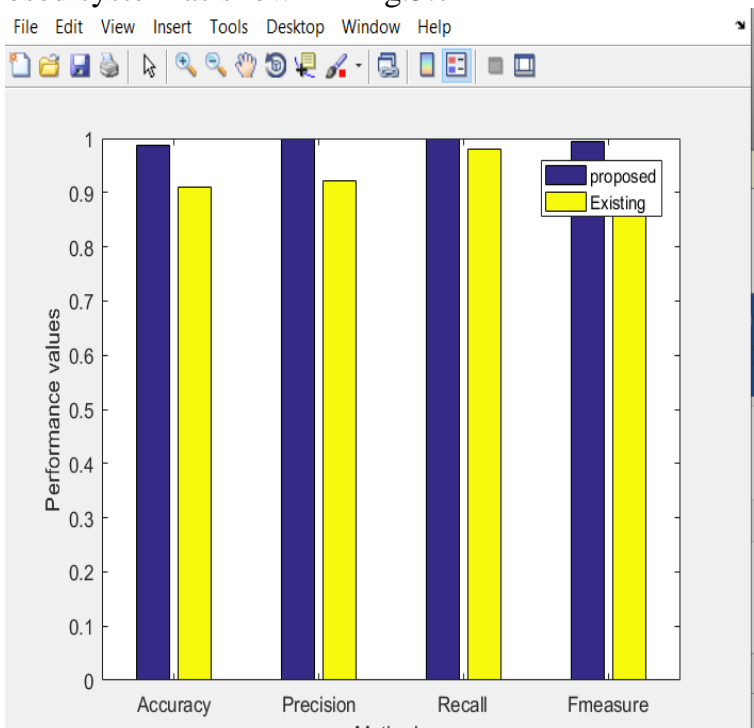

Fig.5.7 Performances values Vs methods

Published By:

Blue Eyes Intelligence Engineering

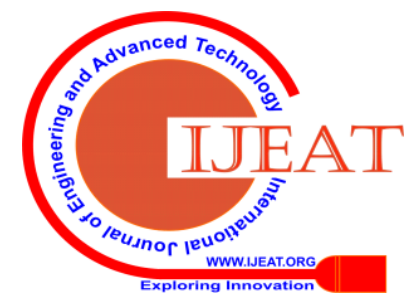




\section{H. Regression Plot}

Fig.5.8

Regression plot of proposed method as shown in

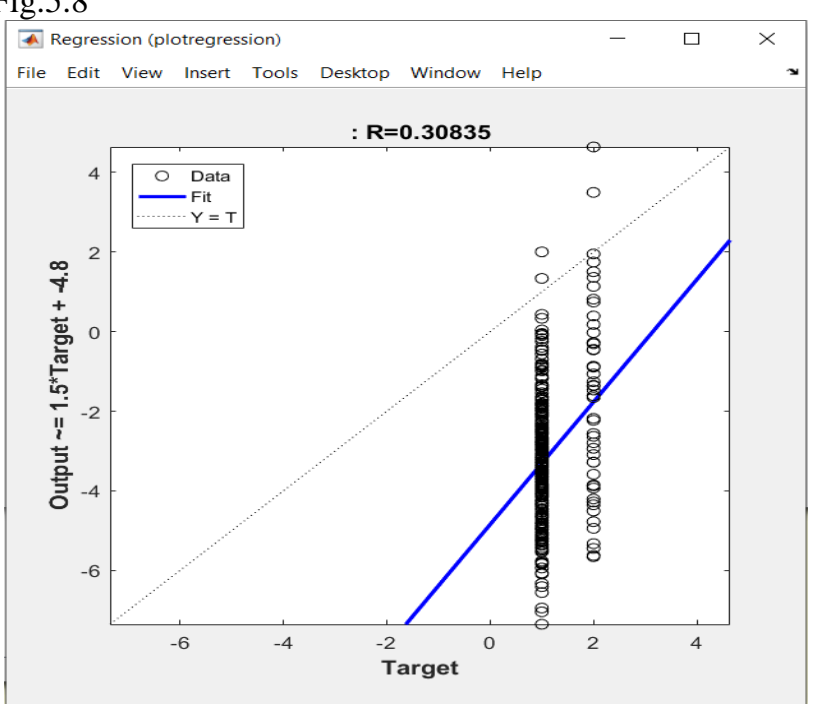

Fig.5.8 Regression Plot

\section{CONCLUSION}

In this method, endomicroscopy image recognition using ensemble neural networks (ENN) was use. The poor-quality of image can produce noise data into the training set which may disturb the final accuracy. To improve the image pixel balancing, contrast- limited adaptive histogram equalization is used (CLAHE). At the same period ensemble, the neural network is used with the combination of other classifiers to improve the accuracy of the result.

\section{REFERENCES}

1. Y Chang, C Jung, and P Ki, H Song, and J Hwang, "Automatic contrast limited adaptive histogram equalization with dual gamma correction," IEEE Access, vol. 6, pp. 11782-11792, 2018.

2. M Cheng, N J Mitra, X Huang, P.H.S. Torr, and S M Hu, "Global contrast based salient region detection," IEEE Trans. Pattern Anal Mach. Intel., vol. 37, no. 3, pp. 569-582, Mar. 2015.

3. S. Coar, G. Donatiello, V. Bogorny, C. Garate, L. O. Alvares, and F. Bremond, "Toward abnormal trajectory and event detection in video surveillance," IEEE Transactions on Circuits and Systems for Video Technology, vol. 27, no. 3, pp. 683-695, March 2017.

4. K. Gu, G. Zhai, X. Yang, W. Zhang, and C. W. Chen, "Automatic contrast enhancement technology with saliency preservation," IEEE Transactions on Circuits and Systems for Video Technology, vol. 25, no. 9, pp. 1480-1494, Sept 2015.

5. A. Heindel, E. Wige, and A. Kaup, "Low-complexity enhancement layer compression for scalable lossless video coding based on hevc," IEEE Transactions on Circuits and Systems for Video Technology, vol. PP, no. 99, pp. 1-1, 2016.

6. Y. H. Shiau, P. Y. Chen, H. Y. Yang, and S. Y. Li, "A low-cost hardware architecture for illumination adjustment in real-time applications," IEEE Transactions on Intelligent Transportation Systems, vol. 16, no. 2, pp. 934-946, Apr 2015.

7. S. W. Kim, B. D. Choi, W. J. Park, and S. J. Ko, "2d histogram equalization based on the human visual system," Electronics Letters, vol. 52, no. 6, pp. 443-445, 2016.

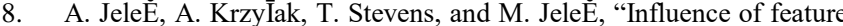
set reduction on breast cancer malignancy classification of fine-needle aspiration biopsies," Computers in Biology and Medicine, vol. 79, pp. 80-91, 2016

9. S. Mittal, H. Kaur, N. Gautam, and A.K. Mantha, "Biosensors for breast cancer diagnosis: A review ofreceptors, bio-transducer and signal amplification strategies," Biosensors and Bioelectronics, vol. 88, pp. 217-231, 2017.

10. Y. Tang, Y. Wang, M.F. Kiani, and B. Wang, "Classification, Treatment Strategy, and Associated Drug Resistance in Breast Cancer," Clinical Breast Cancer, vol. 16, no. 5, pp. 335-343, 2016.

\section{AUTHORS PROFILE}

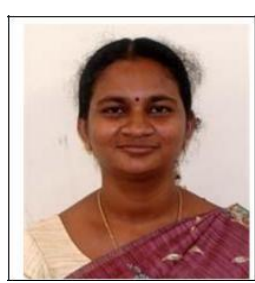

Kalaivani.N, is an assistant professor in Electronics and Communication Engineering at Sri Krishna College of Engineering and Technology, Coimbatore, Tamil Nadu, India. She is having 15 years of teaching experience. She currently works on Medical Image Processing using Deep Learning Algorithms.

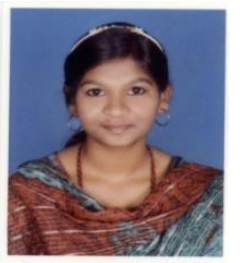

Kani Mozhi.N, currently pursuing a Master's degree in Engineering in the communication system stream under ECE branch at Sri Krishna College of Engineering and Technology. Have published a paper entitled "A patient Monitoring in Ambulance by using Internet Protocol" in ejournal Volume 7, Issue 1 0n March 2019 in International Journal of Scientific Research \& development. Additionally, have presented the paper entitled "Animal footprint Identification for safety purpose" in the second International Conference on "Recent Advances in Engineering and Technology-ICRAET 2019" in February 2019.

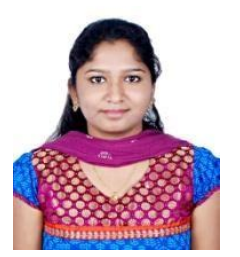

Kanimozhi.M, currently pursuing a Master's degree in Engineering in the communication system stream under ECE branch at Sri Krishna College of Engineering and Technology. Have published a paper entitled "Cognitive Technology in Various Applications" in e-journal Volume 7, Issue 1 0n March 2019 in International Journal of Scientific Research \& development. Additionally, have presented the paper entitled "Application of Selective Region Growing Algorithm in Lung Nodule Segmentation" in $2018^{\text {th }}$ fourth International Conference on Devices, Circuits and Systems (ICDCS)

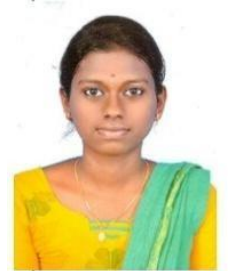

Kalieswari.S, currently pursuing a Master's degree in Engineering in the communication system stream under ECE branch at Sri Krishna College of Engineering and Technology. Have published a paper entitled "A patient Monitoring in Ambulance by using Internet Protocol" in ejournal Volume 7, Issue 1 0n March 2019 in International Journal of Scientific Research \& development. Additionally, have presented the paper entitled "Animal footprint Identification for safety purpose" in the second International Conference on "Recent Advances in Engineering and Technology-ICRAET 2019" in February 2019.

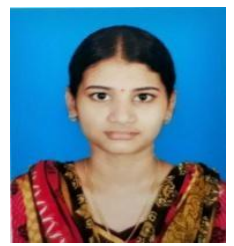

Kuralarasi.R, currently pursuing a Master's degree in Engineering in the communication system stream under ECE branch at Sri Krishna College of Engineering and Technology. Have published a paper entitled "A patient Monitoring in Ambulance by using Internet Protocol" in ejournal Volume 7, Issue 1 0n March 2019 in International Journal of Scientific Research \& development. Additionally, have presented the paper entitled "Animal footprint Identification for safety purpose" in the second International Conference on "Recent Advances in Engineering and Technology-ICRAET 2019" in February 2019.

Published By:

Blue Eyes Intelligence Engineering \& Sciences Publication

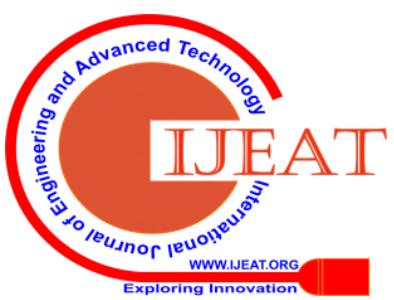

